\title{
Standardization on Particle Size Distribution Measurement of Nanomaterials
}

\author{
Kazuhiro Yamamoto
}

National Institute of Advanced Industrial Science and Technology (AIST), Tsukuba, Japan

Industrial applications of nanomaterials have recently been reported in many fields. Regulation of nanomaterials is being discussed by the Organization for Economic Co-operation and Development (OECD). The European Union (EU) announced their definition of nanomaterial in 2012. According to the EU definition, nanomaterial means a natural, incidental or manufactured material containing particles, in an unbound state or as an aggregate or as agglomerate and where, for $50 \%$ or more of the particles in the number size distribution, one or more external dimensions is in the size range $1 \mathrm{~nm}-100$ $\mathrm{nm}$. They also announced that the particle size of the primary particles in agglomerates or aggregates should be those that are considered. For regulatory purposes, it is necessary to measure the size distribution of nanoparticles based on the particle number concentration. The European Food Safety Authority (EFSA) recommended using at least two different analytical methods to identify nanomaterials for the EU regulation, one of which should be electron microscopy [1]. Transmission electron microscope (TEM) is a most useful technique that can provide precise information on the shape and size of the primary nanoparticles. Standardization on particle size measurement is performed Technical Committee (TC) 229 of International Organization for Standardization (ISO). Scope of ISO/TC229 is standardization in the field of nanotechnologies. In this study, standardization of particle size distribution measurement of nanomaterials by using TEM in ISO/TC229 is introduced.

Interlaboratory comparison (ILC) tests of particle size distribution measurement on mono-dispersed nanoparticles, nanorods, and aggregates were examined in ISO/TC229. Gold nanoparticle was selected as a model material of mono-dispersed case. Maximum and minimum Feret diameter, and equivalent circle diameter (ECD) calculated from area of TEM image were measured. Gold nanorod was model material of anisotropic shaped material. Shaped factor and ECD were measured. In case of the bimodal size distribution, mixture of two kinds of silica nanoparticles was examined. Most of industrial nanoparticles are aggregated. Carbon black (CB) and titanium dioxide $\left(\mathrm{TiO}_{2}\right)$ were selected as model materials of aggregated cases. TEM sample preparation is very important because nanoparticles are easy to aggregate in preparation. In this ILC test, chairpeople of each ILC prepare TEM specimens and distribute to participants of ILC.

These ILC tests are now in progress, and data gathering by each chairperson is performing. In this presentation, some results of ILC by authors are shown. In ILC test of gold nanorod, two kinds of specimen are examined. Figure 1(a) and (b) shows TEM images of two gold nanorod specimens, named \#1 and \#2. Two specimens look like similar, and the difference of two is not clear. Size analysis of these gold nanorod is examined statistically. TEM images are recorded by using slow scan CCD camera at the resolution of $0.154 \mathrm{~nm} /$ pixel. Area of projection images of each nanorods, maximum, and minimum Feret diameter are measured by using Image-J software. Equivalent circular diameter (ECD) is calculated from projection area, and figures 2(a) and (b) show histogram and cumulativetive distribution of ECD, respectively. Number of counted particles is 753 for specimen \#1, and 840 for specimen \#2. ECD distributions are in ranging $20 \mathrm{~nm}$ and $43 \mathrm{~nm}$. Median diameter (d50) is $36.5 \mathrm{~nm}$ for specimen \#1, and $37.5 \mathrm{~nm}$ for specimen \#2. Shape factor is defined as the ratio of maximum and minimum Feret diameters, and figure 3 shows cumulative distribution of shape factor. Shape distribution of specimen \#2 
is wider than that of specimen \#1. Optical functions of gold nanorod are strongly depends on their size and shape, therefore the evaluation and standardization to measure size and shape is important.

\section{References}

[1] E.A.J. Bleeker et al, RIVM Letter report 601358001(2012).
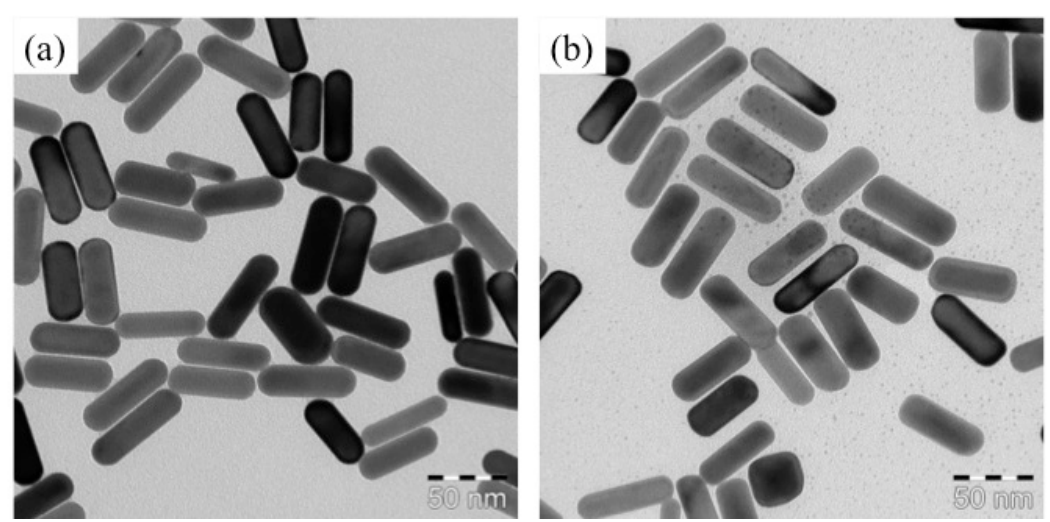

Figure 1. Typical TEM images of gold nanorod. Specimen \#1 (a), and specimen \#2 (b).
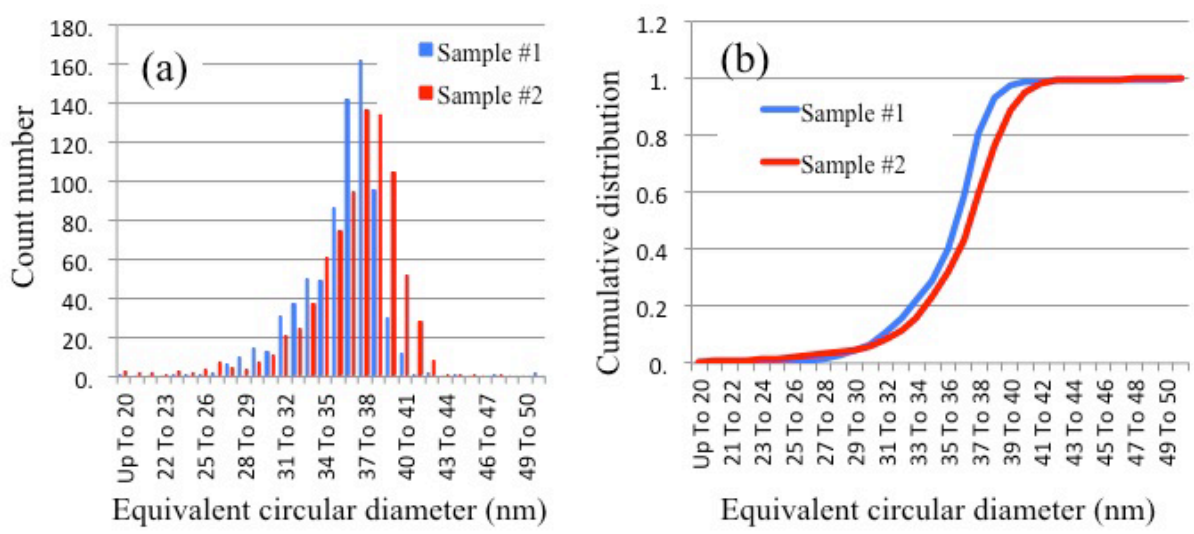

Figure 2. Equivalent circular diameter distribution of gold nanorod specimens (a), and cumulative distribution (b).

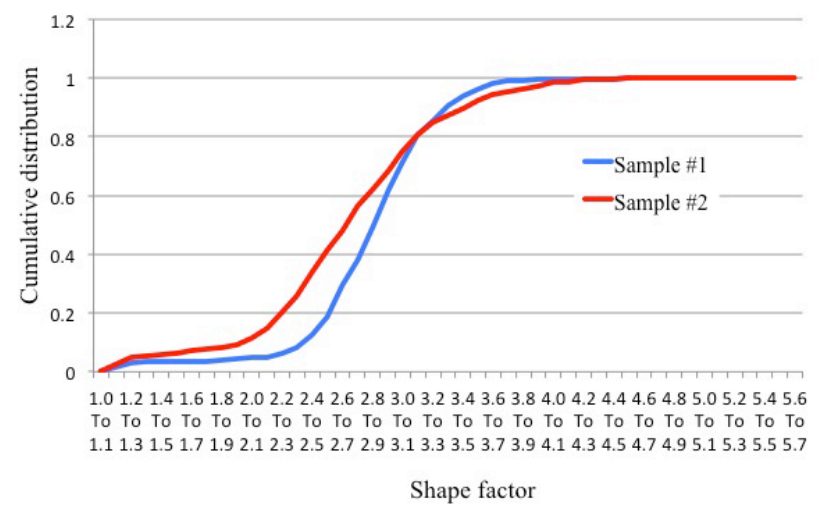

Figure 3. Cumulative distribution of shape factor. 